\title{
Anatomical and Functional Outcome of Pars Plana Vitrectomy and Subretinal Recombinant Tissue Plasminogen Activator for a Macular Subpigment Epithelial Haemorrhage
}

\author{
Konstantinos Fotis Raquel Garcia-Cabrera Mya Ohn Aman Chandra \\ Southend University Hospital NHS Foundation Trust, Westcliff-on-Sea, UK
}

Dear Editor

We read the recent article by Waizel et al. [1] with great interest. They concluded that vitrectomy combined with subretinal recombinant tissue plasminogen activator (rtPA) injection and gas or air tamponade has a strong functional and anatomical effect on both subneuroretinal haemorrhage $(\mathrm{SRH})$ and combined subneuroretinal and sub-retinal pigment epithelial (RPE) haemorrhage $(\mathrm{CH})$ and also seems to slightly improve the anatomical outcome in subpigment epithelial haemorrhage (SPH) [1].
We congratulate the authors for their valuable study and their novel approach to the analysis of the treatment of submacular haemorrhages based on anatomic location. We feel we can contribute to this ongoing discussion with our own experience of an interesting case. Various treatment methods are currently in use for submacular haemorrhages, but recent trends in the literature suggest that more surgeons are using vitrectomy over pneumatic displacement despite lack of evidence to fully sup- port one method over another [2-4]. Our case involves an 88-year-old female patient, with a previous history of bilateral neovascular age-related macular degeneration treated with bilateral intravitreal injections of aflibercept, who presented with a 4-day history of reduction of right vision. Right visual acuity was $1.20 \log$ MAR. Clinical and OCT examination demonstrated a submacular haemorrhage with combined subneuroretinal and RPE components (CH) (Fig. 1, 2). She underwent pneumatic
1

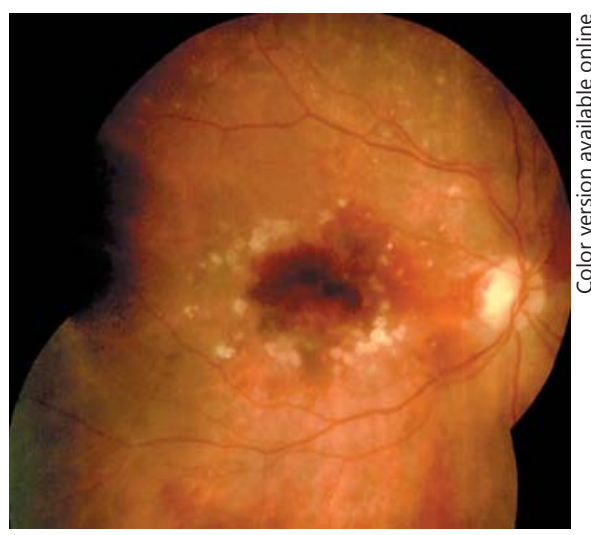

Fig. 1. Fundus photograph at presentation. Fig. 2. OCT at presentation.
2

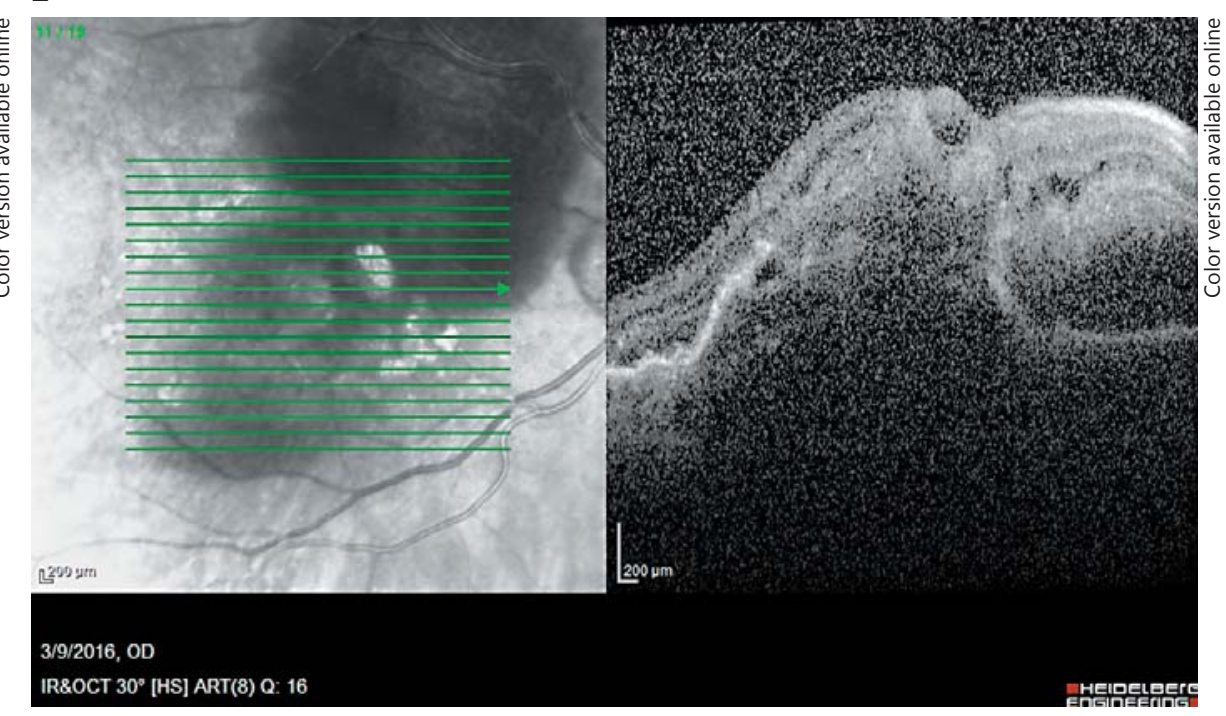

\section{KARGER}

(C) 2017 S. Karger AG, Basel

E-Mail karger@karger.com www.karger.com/oph
Konstantinos Fotis

Southend University Hospital NHS Foundation Trust Prittlewell Chase

Westcliff-on-Sea SS0 ORY (UK)

E-Mail konstantinosfotis@nhs.net 
Fig. 3. OCT 5 days post intravitreal $\mathrm{rtPA}$ and $100 \%$ SF6 gas.

Fig. 4. Fundus photograph 1 week post 23-G pars plana vitrectomy, subretinal rtPA, and 25\% SF6 gas.

Fig. 5. OCT 1 week post $23-\mathrm{G}$ pars plana vitrectomy, subretinal rtPA, and 25\% SF6 gas.
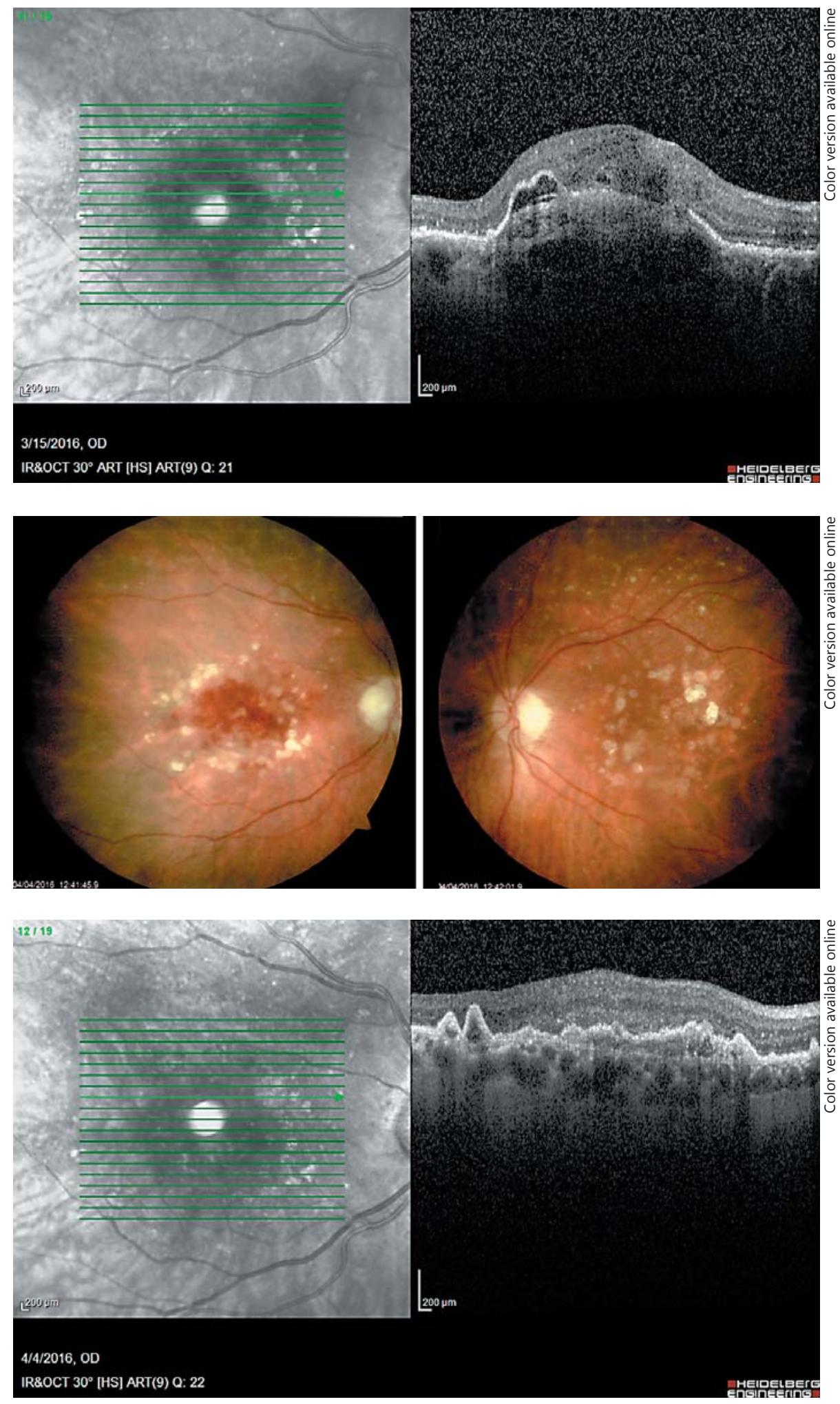

Vitrectomy and Subretinal rtPA for a Macular SPH
Ophthalmologica 2017;238:106-108 DOI: $10.1159 / 000475891$ 
displacement with intravitreal rtPA (50 $\mu \mathrm{g} / 0.1 \mathrm{~mL}$ ) and $0.4 \mathrm{~mL}$ of $100 \%$ sulphur hexafluoride (SF6) gas. This displaced the SRH and resulted in visual acuity improvement to $1.00 \log$ MAR 5 days later (Fig. 3). The SPH unfortunately persisted. Further treatment was decided and she underwent 23-G pars plana vitrectomy, subretinal rtPA $(40 \mu \mathrm{g} / 0.4 \mathrm{~mL})$, and $25 \%$ SF6. This resulted in reduction of the SPH (Fig. 4, 5), with visual acuity improvement to $0.60 \mathrm{log}$ MAR 1 week postoperatively. We can only hypothesise as to the effect of treatment to the SPH, as there are no signs that the haemorrhage was displaced as seen when treating $\mathrm{SRH}$, and the $\mathrm{rtPA}$ is still injected

\section{References}

1 Waizel M, Todorova MG, Kazerounian S, Rickmann A, Blanke BR, Szurman P: Efficacy of vitrectomy combined with subretinal recombinant tissue plasminogen activator for subretinal versus subpigment epithelial versus combined hemorrhages. Ophthalmologica 2016;236:123-132.

2 Fassbender JM, Sherman MP, Barr CC, Schaal S: Tissue plasminogen activator for subfoveal hemorrhage due to age-related macular degeneration: comparison of 3 above the RPE. Diffusion of the medication through the RPE and absorption of the fibrinolysis products by the choroidal circulation seems to be a possible explanation. Alternatively, the observed anatomical and functional improvement could also have occurred after our first therapeutic intervention with pneumatic displacement [5], or even as part of the natural history of the condition. Finally, the role of time from presentation to treatment also must not be understated in our favourable outcome. We feel our case is an example of both the effectiveness of subretinal rtPA in $\mathrm{CH}$ and the fact that a functional improvement is also possible in cases with SPH.

treatment modalities. Retina 2016;36:18601865.

3 Bell JE, Shulman JP, Swan RJ, Teske MP, Bernstein PS: Intravitreal versus subretinal tissue plasminogen activator injection for submacular hemorrhage. Ophthalmic Surg Lasers Imaging Retina 2017;48:26-32.

4 de Jong JH, van Zeeburg EJ, Cereda MG, van Velthoven ME, Faridpooya K, Vermeer KA, van Meurs JC: Intravitreal versus subretinal administration of recombinant tissue plas-

\section{Statement of Ethics}

Subjects have given their informed consent for any procedures carried out. This is not a study and no protocol has been submitted for approval to our institute's ethics committee.

\section{Disclosure Statement}

There are no conflicts of interest for any of the authors. minogen activator combined with gas for acute submacular hemorrhages due to agerelated macular degeneration: an exploratory prospective study. Retina 2016;36:914-935.

5 Nayak S, Padhi T R, Basu S, Das T: Pneumatic displacement and intravitreal bevacizumab in management of sub-retinal and sub-retinal pigment epithelial hemorrhage at macula in polypoidal choroidal vasculopathy (PCV): rationale and outcome. Semin Ophthalmol 2015;30:53-55. 